\title{
Co-channel interference management using elCIC/FelCIC with coordinated scheduling for the coexistence of PS-LTE and LTE-R networks
}

Wan Chen, Ishtiaq Ahmad and KyungHi Chang ${ }^{*}$ (D)

\begin{abstract}
In the Republic of Korea, a Long Term Evolution (LTE)-based public safety (PS)-LTE network is being built using 718 728 MHz for uplink and 773 783 MHz for downlink. However, the same bands are also assigned to the LTE-based high-speed railway (LTE-R) network, so great concerns and practical researches on co-channel interference (CCI) management schemes are urgently required. In this paper, performance is analyzed and evaluated by considering the cases of non-RAN (radio access network) sharing and LTE-R RAN sharing by PS-LTE user equipments (UE). Since a train control signal requires high reliability and low latency in order to fulfill its mission-critical service (MCS) requirements, we give higher priority to LTE-R UE during resource allocation under the LTE-R RAN sharing by PS-LTE UEs. In addition, interference management schemes are more effective for the coexistence of PS-LTE and LTE-R networks under RAN sharing environment. In this paper, we utilize enhanced inter-cell interference coordination (elCIC) and further enhanced ICIC (FeICIC) schemes to mitigate the interference from PS-LTE network to LTE-R network while improving the LTE-R eNodeB (eNB) resource utilization by offloading more PS-LTE UEs to LTE-R network. Moreover, a coordinated multipoint (CoMP) transmission scheme is considered among LTE-R eNBs to enhance LTE-R cell edge user performance. By employing FeICIC along with coordinated scheduling (CS) CoMP, the best throughput performance can be achieved under the case of RAN sharing.
\end{abstract}

Keywords: PS-LTE, LTE-R, Coexistence, QoS priority, (F)elCIC, CoMP

\section{Introduction}

In the Republic of Korea, the national disaster safety network project has been started in 2014 [1], which costs over 1.6 billion dollars, and is being built using Long Term Evolution (LTE) for public safety on the $700 \mathrm{MHz}$ frequency band. Frequency bands for next-generation railway network and e-navigation network over marine environment are also assigned to the same $700 \mathrm{MHz}$ bands as the public safety network. It is known that target users of the public safety network are police, firefighters, soldiers, emergency medical workers, and so on, and the LTE-based high-speed railway (LTE-R) network will provide communication services to control trains and for train crews. Because railway wireless

\footnotetext{
* Correspondence: khchang@inha.ac.kr

Department of Electronic Engineering, Inha University, Incheon, Korea
}

communication together with train control has been crucial for the reliability and safety of railway operation, if both networks use the same frequency, great concerns and practical researches on interference management schemes are urgently required.

Radio access network (RAN) sharing is currently considered as a candidate for the coexistence of these two networks [2]. For the coexistence of public safety (PS)LTE and LTE-R networks, which are assumed to use the same spectrum, active RAN sharing should be considered. LTE-R user equipment (UE) usually receives strong downlink (DL) signals from LTE-R eNodeBs (eNBs) due to the short distance to the railway. Under the assumption of very reliable LTE-R network deployment, we only consider LTE-R RAN sharing by PS-LTE UEs.

In the 3rd Generation Partnership Project (3GPP) LTE Rel. 8, inter-cell interference coordination (ICIC) is 
proposed, by which eNBs can communicate via the X2 interface and optimize scheduling for cell edge users. In 3GPP LTE Rel. 10, ICIC is enhanced to better support heterogeneous network (HetNet) deployments, which is eICIC [3]. The major difference is the additional timedomain ICIC, realized through almost blank subframes (ABS). During ABS, no traffic is transmitted except the control signaling and cell specific reference signals to mitigate the interference from macro eNodeBs (MeNB) to the cell edge UEs in small cells, especially the UEs in the cell range expansion (CRE) area $[4,5]$. In 3GPP LTE Rel. 11, further enhanced ICIC (FeICIC) is proposed, which allows traffic data to be transmitted during ABS with relatively low power $[6,7]$.

For the coexistence of PS-LTE and LTE-R networks, due to the different range of coverage and overlapping deployment, the interference management schemes for heterogeneous networks are better to be adopted. In this paper, we consider eICIC and FeICIC as candidates. Since the coverage of PS-LTE eNB is much larger than LTE-R eNBs, ABSs and power-reduced ABSs (PR-ABS) are used to protect the cell edge UEs served by LTE-R eNBs against the interference from PS-LTE eNBs. Simultaneously, CRE is used to offload more PS-LTE UEs to LTE-R eNBs to further enhance the efficiency of resource utilization of LTE-R eNBs to improve system throughput.

A coordinated multipoint (CoMP) is recognized as an effective method to further improve the cell edge users' performance. In Rel. 10 [8], downlink (DL) CoMP includes the possibility of coordination among different points or cells. If inter-eNB coordination is supported, information needs to be signaled among eNBs. There are various types of CoMP schemes: joint transmission (JT), dynamic point selection (DPS), and coordinated scheduling/beamforming (CS/CB). For JT and DPS, the data for an UE should be available at multiple transmission points, but at only one transmission point for CS/ $\mathrm{CB}$. In this paper, CS CoMP is considered for the coexistence of PS-LTE and LTE-R networks.

It should be noted that the LTE-R network can be built in centralized (C)-RAN architecture in 5G. With this assumption, the interference management schemes are also effective. The LTE-R eNBs in our scenario can be regarded as a group of RRUs (remote radio unit) which are connected to one BBU (baseband unit), and the centralized coordinated scheduling algorithm can be executed in the BBU. The combination of eICIC/FeICIC with CoMP can also be used in $5 \mathrm{G}$ ultra dense network. Similarly, eICIC/FeICIC can be used to prevent interference from macrocells to the small cells, while at the same time, CoMP can be applied to a group of small cells [9]. The cooperation among the small cells costs more than the case in our scenario, since LTE-R eNBs are deployed along the train track.
In this paper, a typical scenario for the coexistence of PS-LTE and LTE-R is considered, and we evaluate the popular inter-cell interference management schemes while applying the practical channel model, channel quality indicator (CQI) feedback, and scheduling procedure. After that, the effective interference management techniques are employed while noticing the feasibility, complexity, and the details of how to combine as well as to utilize these techniques are illustrated. The rest of the paper is organized as follows. In section 2, we present the system model used to analyze the co-channel interference (CCI) between the PS-LTE and LTE-R networks. Section 3 compares the cases of non-RAN sharing and LTE-R RAN sharing by PS-LTE UEs, and the interference management schemes are introduced in Section 4. In section 5 , the performance of the interference management schemes is evaluated using system-level simulations (SLS). Finally, section 6 concludes the paper.

\section{System model for the coexistence of PS-LTE and LTE-R networks}

2.1 PS-LTE and LTE-R network deployment

The inter-site distance (ISD) of PS-LTE and LTE-R networks is assumed to be $4 \mathrm{~km}$ [10] and $1 \mathrm{~km}$, respectively. We consider a scenario with one-tier deployment of PS-LTE eNBs and four LTE-R eNBs overlapped with the center site of PS-LTE network, as shown in Fig. 1. The center area is the region of interest (ROI). There are three sectors for each PS-LTE eNB, but only two sectors for each LTE-R eNB to support the coverage over the railway. The LTE-R eNBs are located on the both sides of the track.

\subsection{Channel model}

To calculate the propagation loss for each link, the general equation for the channel gain is given in Eq. (1):

$$
G=\text { Antenna_Gain-PathLoss-Shadowing-Fading }
$$

The Hata model is widely used for path loss [11], which can support the carrier frequency range from 150 to $1500 \mathrm{MHz}$. In this paper, the Hata rural model is considered and given as:

$$
\begin{aligned}
L(R)=69.55 & +26.16 \log _{10}(f)-13.82 \log _{10}\left(h_{b}\right) \\
& +\left[44.9-6.55 \log _{10}\left(h_{b}\right)\right] \log _{10}(R) \\
& -4.78\left(\log _{10}(f)\right)^{2}+18.33 \log _{10}(f)-40.94
\end{aligned}
$$

where $R$ is the distance between the eNB and UE in $\mathrm{km}$, $f$ is the carrier frequency in $\mathrm{MHz}$, and $h_{b}$ is the base station antenna height above ground in meters.

Shadowing is modeled by using log-normal distribution with a mean of $0 \mathrm{~dB}$ and standard deviation of $6 \mathrm{~dB}$ [12]. For the paths from the same eNBs to two UEs at 


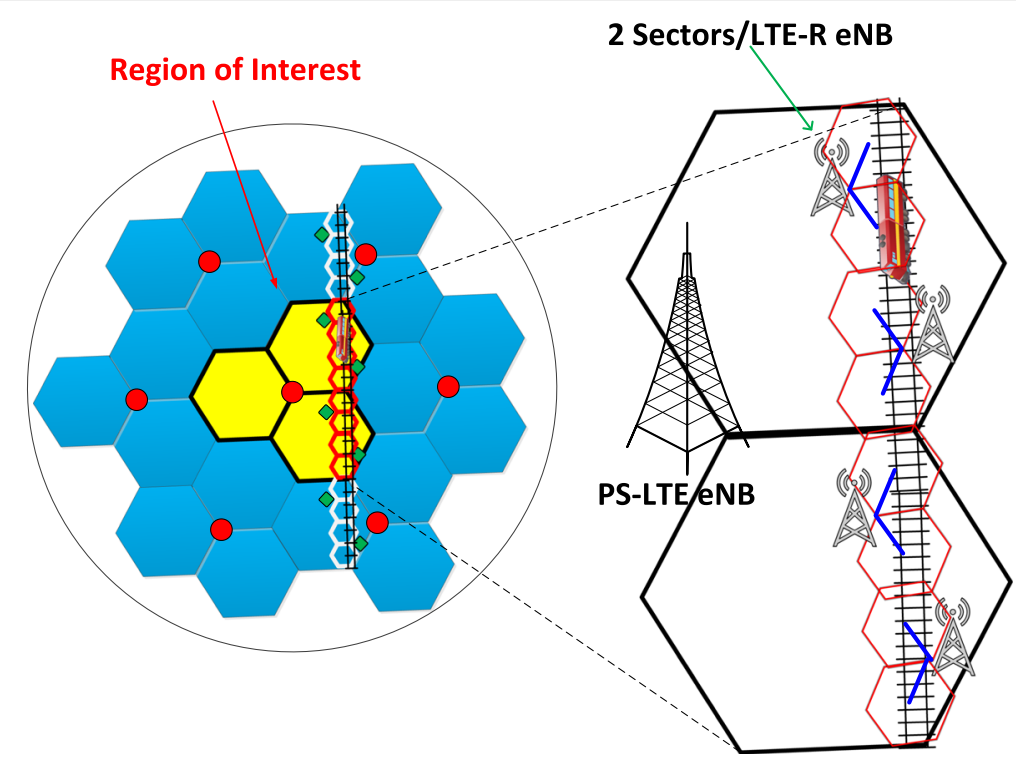

Fig. 1 PS-LTE and LTE-R network deployment

different positions, spaced by a distance $x$, the shadowing correlation coefficient is $r(x)=e^{-\alpha x}$. In addition, the inter-site correlation coefficient is 0.5 .

Fast fading refers to rapid variation of the signal levels due to multipath transmission. In this paper, fast fading is generated according to the D1 and D2a scenarios supported by Winner II [13] for PS-LTE UEs with low mobility and LTE-R UEs with high mobility, respectively.

3D antenna pattern is widely used [11], which considers horizontal cut and vertical cut of antenna gain as follows:

$$
\left\{\begin{array}{l}
\mathrm{A}_{\mathrm{V}}\left(\theta_{\mathrm{V}}\right)=\min \left[12\left(\frac{\theta_{\mathrm{V}}-\theta_{\text {etilt }}}{\theta_{3 \mathrm{~dB}}}\right)^{2}, \mathrm{SLA}_{\mathrm{v}}\right] \\
\mathrm{A}_{\mathrm{H}}\left(\varphi_{\mathrm{H}}\right)=\min \left[12\left(\frac{\varphi_{\mathrm{H}}}{\varphi_{3 \mathrm{~dB}}}\right)^{2}, A_{m}\right]
\end{array}\right.
$$

where $A_{V}$ and $A_{H}$ are the vertical and horizontal cut, respectively. $\theta_{\mathrm{V}}$ and $\varphi_{\mathrm{H}}$ are the angles between the sector antenna direction and the eNB-to-UE transmission path direction on the vertical plane and horizontal plane, respectively. $\varphi_{3 \mathrm{~dB}}$ and $\theta_{3 \mathrm{~dB}}$ are the $3-\mathrm{dB}$ horizontal and vertical beam widths, respectively, and $A_{m}$ and $S L A_{v}$ are backward attenuation and side lobe vertical attenuation, respectively. $\mathrm{A}(\theta, \varphi)$ is the total attenuation. The antenna gain should be calculated as:

AntennaGain $=$ max $\_$AntennaGain $-\min \left[\mathrm{A}_{\mathrm{V}}\left(\theta_{V}\right)+\mathrm{A}_{\mathrm{H}}\left(\varphi_{\mathrm{H}}\right), A_{m}\right]$

Typical values for these parameters are $\varphi_{3 \mathrm{~dB}}=70^{\circ}, A_{m}=$ $20 \mathrm{~dB}, \theta_{3 \mathrm{~dB}}=10^{\circ}, \quad \mathrm{SLA}_{\mathrm{V}}=20 \mathrm{~dB}$, and $\theta_{\text {etilt }}=\left(0^{\circ}, 15^{\circ}\right)$.

\subsection{Abstraction of physical layer}

The abstraction of physical (PHY) layer is meant to obtain the block error rate (BLER) for each transport block (TB) under the corresponding modulation and coding scheme (MCS) to calculate UE throughput. In LTE system, there is a loop for the adaptive modulation and coding (AMC) scheme in DL transmission, which requires UE feedback of the channel quality indicator (CQI) index. Each CQI index corresponds to a certain MCS. By using various MCS levels, different spectral efficiencies can be achieved [14]. In addition, the MCS level is one factor in deciding the BLER for the corresponding $\mathrm{TB}$ as well as the signal-to-interference-plusnoise ratio (SINR). To get the BLER in SLS without carrying out real signal processing, we use the curves obtained by link-level simulation (LLS) under an additive white Gaussian noise (AWGN) channel with respect to 15 MCS levels. To predict the BLER under a fading channel, AWGN-equivalent SINR is required. In this paper, mutual information-based exponential SINR mapping (MIESM) [15] is used to map the SINRs of multiple subcarriers assigned to the TB to the AWGN-equivalent effective SINR.

\section{Analysis on co-channel interference for the coexistence of LTE-R and PS-LTE networks}

\subsection{LTE-R and PS-LTE network coexistence without RAN} sharing

For the scenario without RAN sharing illustrated in Fig. 2. PS-LTE UEs are not allowed to access LTE-R eNBs, which have smaller coverage overlapped with the PS-LTE network. This is similar to the coexistence of macrocells and closed subscriber group (CSG) femtocells, 


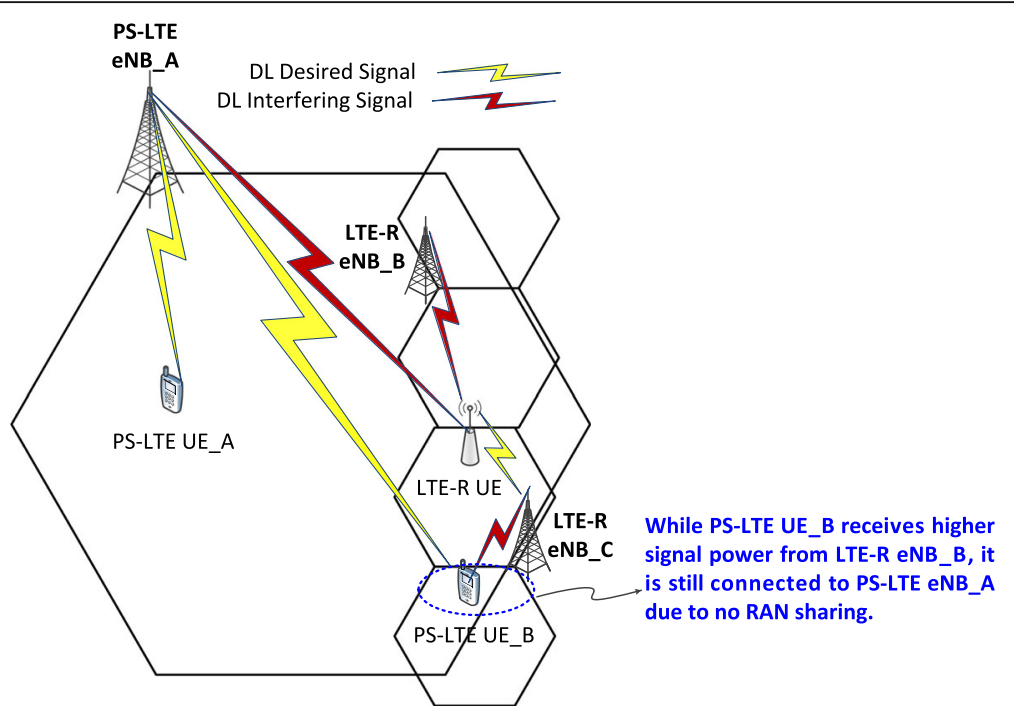

Fig. 2 Coexistence of PS-LTE and LTE-R networks without RAN sharing

in which the low-power nodes (LPN) only allow access by a group of UEs. However, LTE-R eNBs are high-power nodes but with a limited coverage along the track, which results in more severe co-channel interference, especially when LTE-R eNBs are located at the cell edge of PS-LTE network, as shown in Fig. 2. For the PS-LTE UE_B at the edge of PS-LTE coverage, which simultaneously receives a relatively low-power desired signal, the interference power from the LTE-R eNBs is really high because the UE is also in the center coverage of LTE-R network.

The power setting is already specified in [6] for eICIC/FeICIC schemes in order to protect the macro UEs that are close to the CSG femtocells by decreasing the transmission power of the femtocells, because femto eNBs are LPNs and are usually deployed by users for limited service requirements, which gives the small cells lower priority. Hence, it is rational to protect macro UEs by reducing the transmission power of femto eNBs. However, both PS-LTE and LTE-R networks are high-power nodes deployed by operators. In addition, reducing the power of LTE-R eNBs will impact the reliability of the LTE-R network and cause outage in service so the eICIC/FeICIC schemes are not effective under the environment without RAN sharing.

\subsection{LTE-R RAN sharing by PS-LTE UEs}

Instead of considering LTE-R eNBs as sources of high interference, they can be considered as eNBs to enhance the cell edge coverage of PS-LTE network by active RAN sharing. Under the RAN sharing environment, PSLTE UEs can connect with LTE-R eNBs, which reduces the co-channel interference and boosts the resource utilization of LTE-R eNBs. However, LTE-R UE is moving along the track and usually receives higher power from LTE-R eNBs, so it is not necessary for PS-LTE network to support RAN sharing for LTE-R UEs. In this paper, only LTE-R RAN sharing by PS-LTE UEs is considered.

\subsubsection{Scheduling for LTE-R RAN sharing by PS-LTE UES}

In the RAN sharing case, we consider predefined rules for scheduling. Since the downlink transmission for LTE-R UE requires low latency and high reliability, it is necessary to assign the best resources to the LTE-R UE. Thus, in order to fulfill the LTE-R mission-critical service requirements, we always give higher priority to LTE-R UE during resource allocation. Figure 3 shows the scheduling process for LTE$R$ eNBs that offer RAN sharing to PS-LTE UEs, while PS-LTE eNB schedule their UEs based on proportional fair scheduling.

\section{4 elCIC/FelCIC schemes with coordinated scheduling under the coexistence of PS-LTE and LTE-R networks}

In this section, we introduce effective interference mitigation schemes under RAN sharing environment to improve the LTE-R UE channel quality, meanwhile further improving PS-LTE UE performance, including both reliability and throughput. It should be noted that our scenario of PS-LTE and LTE-R network coexistence is a unique HetNet scenario with overlapped macrocells, and both of them are deployed by operators. Considering the difference in cell sizes and that LTE-R eNBs can offload 


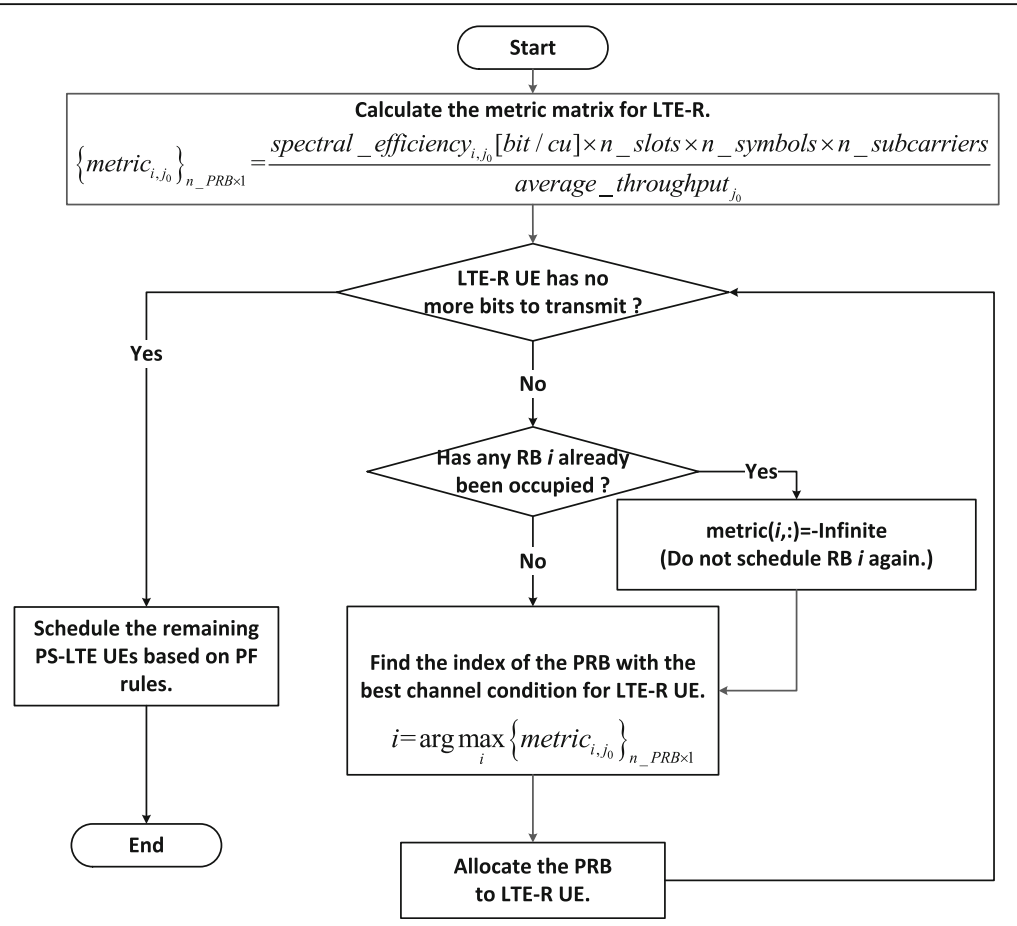

Fig. 3 LTE-R eNB scheduling with RAN sharing for PS-LTE UES

PS-LTE UEs using RAN sharing, this scenario is similar to the HetNet scenario of macro and picocells, in which UEs can connect with both the two types of eNBs and pico eNBs with smaller coverage are supposed to offload macro UEs [8]. However, there is an obvious difference, which is that there are two types of UEs in our scenarios, PS-LTE UEs can be distributed anywhere and considered as normal UEs but LTE-R UE is moving along the track located between the LTE-R eNBs. Targeting this scenario with RAN sharing, time-domain eICIC and FeICIC schemes can be employed to restrain the interference from PS-LTE eNBs to LTE-R network. As for the LTE-R UE along the track, it usually receives higher interference power from neighbor LTE-R eNBs than that from PS-LTE eNBs. To mitigate this interference, CS CoMP is considered between the neighbor LTE-R eNBs.

\subsection{General notations and scenario description}

The general notations of the considered network are given as follows:

- M: set of PS-LTE cells.

- $K$ : set of LTE-R cells.

- $U$ : set of UEs, which can be divided into two sets, $U_{M}$ and $U_{K}$, served by PS-LTE eNBs and LTE-R eNBs, respectively.

- $U_{M}$ : set of PS-LTE UEs served by PS-LTE cells.
- $U_{K}$ : set of UEs served by LTE-R eNBs; can be both LTE-R and PS-LTE UEs.

- $U_{C K}$ : set of cell center UEs of LTE-R cells.

- $U_{E K}$ : set of CRE UEs of LTE-R eNBs will only be scheduled during ABSs.

- $U_{C M}$ : set of cell center UEs of PS-LTE eNBs, which will be scheduled during PR-ABSs.

- $U_{E M}$ : set of the rest of the UEs of PS-LTE eNBs, which will only be scheduled during normal subframes when FeICIC is applied.

- $n$ : index of the physical resource block (PRB) for $10 \mathrm{MHz}$ bandwidth (50 PRBs).

- $G_{m, u}^{n}$ : channel gain from PS-LTE cell $m$ to UE $u$ on PRB $n$.

- $G_{k, u}^{n}$ : channel gain from LTE-R cell $k$ to UE $u$ on PRB $n$.

- $I_{i, u}^{n}$ : interference to UE $u$ from neighbor PS-LTE cell $i$ on PRB $n$.

- $I_{j, u}^{n}$ : interference to UE $u$ from neighbor LTE-R cell $j$ on PRB $n$.

- $\eta$ : thermal noise per RB, including the UE noise figure.

- $\mathrm{RB}_{p}^{k}$ : set of RBs, scheduled by LTE-R cell $k$ during ABSs, which are protected resources.

- $\mathrm{RB}_{\mathrm{np}}^{k}$ : set of RBs, scheduled by LTE-R cell $k$ during normal subframes, which are non-protected resources.

- $\mathrm{RB}_{\mathrm{fp}}^{m}$ : set of RBs, scheduled by PS-LTE cell $k$ during normal subframes by using full transmission power. 


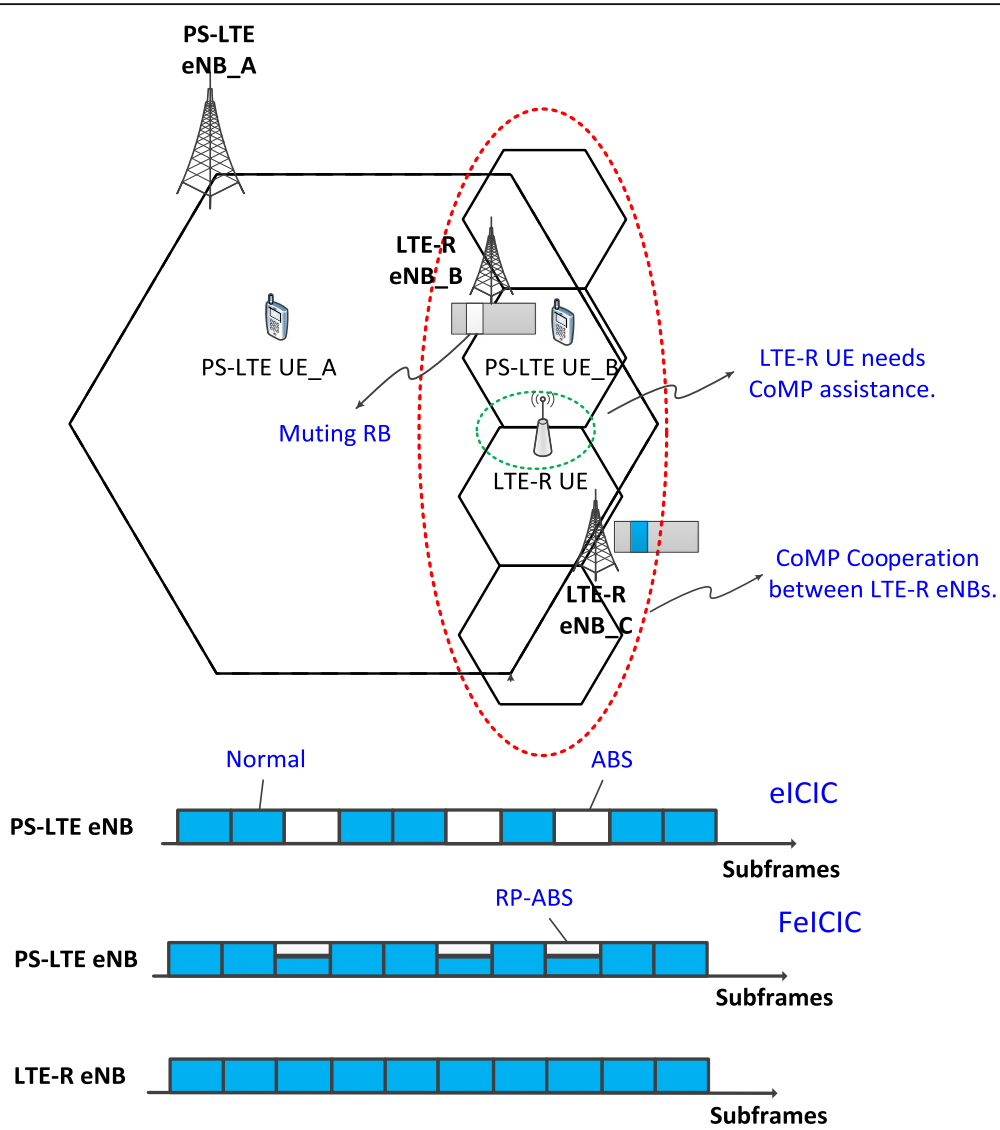

Fig. 4 Scenarios 3 and 4: LTE-R RAN sharing by PS-LTE with elCIC/FelCIC and CS COMP

- $\mathrm{RB}_{\mathrm{rp}}^{m}$ : set of RBs, scheduled by PS-LTE cell $k$ during normal subframes by using reduced transmission power.

- $r_{u}^{n}$ : rate that can be achieved by RB $n$ while allocated to UE $n$.

- $R$ : total throughput of the system, in bit/second.

Figure 4 shows the scenarios of RAN sharing with interference management schemes of eICIC/FeICIC with CS CoMP. For eICIC, during ABSs, no data is transmitted from PS-LTE eNB to avoid interference towards PSLTE UEs served by the LTE-R eNB and the LTE-R UE. By using FeICIC, data is transmitted during powerreduced ABSs for the center PS-LTE UEs while keeping the interference to the UEs supported by LTE-R eNBs at a relatively low level. However, the introduction of ABS/ PR-ABS will only benefit the cell edge UEs of LTE-R eNBs. To protect the PS-LTE UEs which are getting high interference from LTE-R eNBs, CRE is introduced by adding a positive bias to the RSRPs of LTE-R eNBs to offload them into the LTE-R eNBs and then they can benefit from ABSs/PR-ABSs. Therefore, the criterion for serving cell selection is given by as follows:

$$
\text { Serving_eNB_ID }=\underset{i \in(M \cup K)}{\arg \max }\left\{\operatorname{RSRP}_{i}+\operatorname{bias}_{i}\right\}
$$

where $\operatorname{bias}_{i}=0 \mathrm{~dB}$ if $i \in M$ and bias $_{i}>0 \mathrm{~dB}$ if $i \in K$.

\section{2 elCIC/FelCIC schemes between PS-LTE and LTE-R eNBs 4.2.1 elCIC/FelCIC operation rules}

When PS-LTE eNBs are under the condition of high load, LTE-R coverage can be extended to allow more PS-LTE UEs to be offloaded and then the remaining PSLTE UEs with better channel quality can be well served by PS-LTE eNBs. In this regard, the offloaded UEs will suffer severe co-channel interference from the PS-LTE eNBs. Thus, ABS/PR-ABS is applied in order to mitigate the interference to the offloaded UEs.

Figure 5a shows the flow chart of the combination of eICIC/FeICIC and CS CoMP, and the details of CS CoMP will be introduced in section 4.3. The overall procedure of eICIC/FeICIC can be divided into three main parts: cell selection with CRE, UE identification, and scheduling. In flow chart (a), the threshold for RAN sharing is the bias used to extend the coverage of LTE-R eNBs. Flow charts (b) and (c) show the rules of UE 

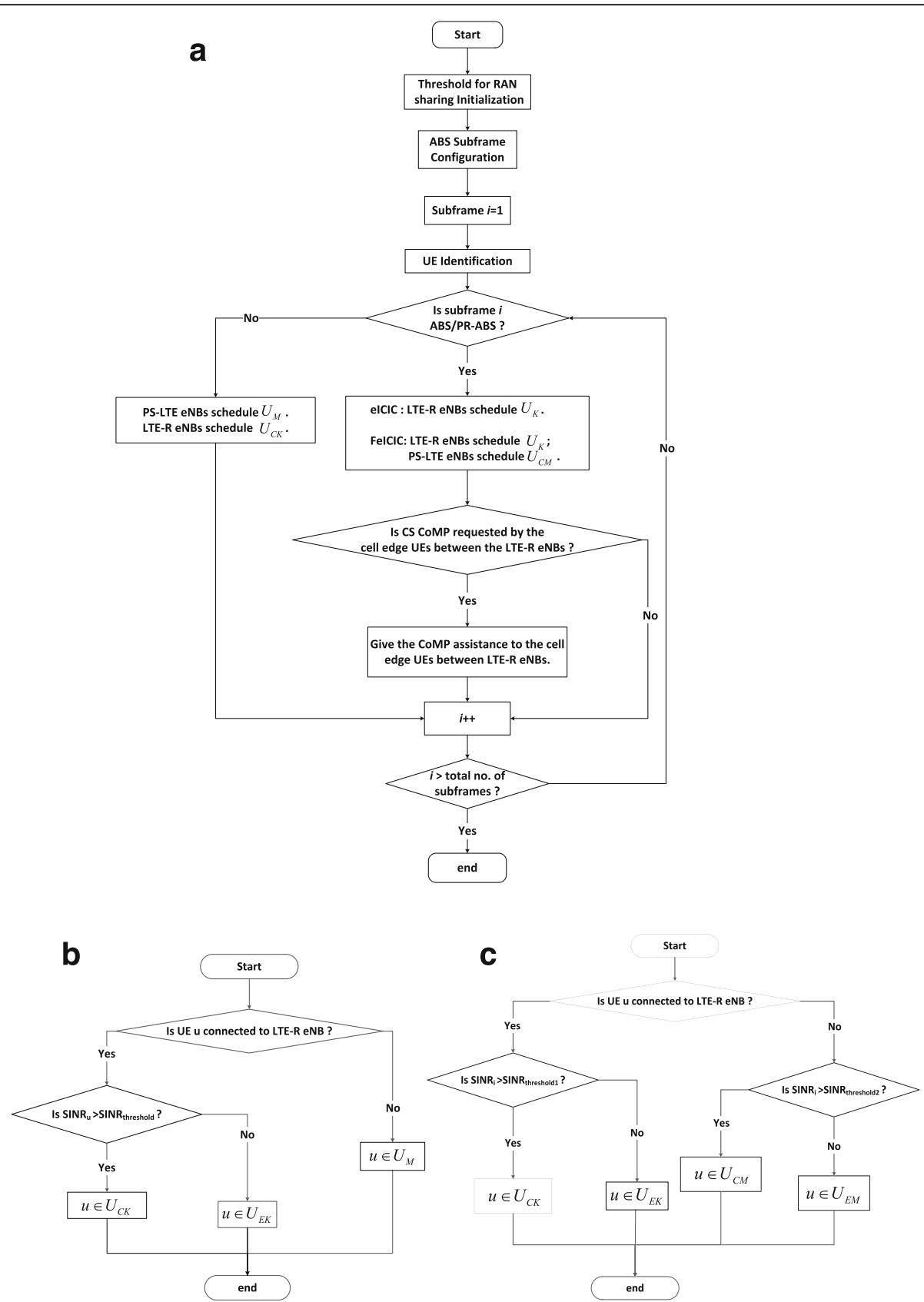

Fig. 5 Flow charts for the procedures of elCIC/FeICIC and CS CoMP: a overall flow chart, b UE identification for elCIC, c UE identification for FelClC

classification for eICIC and FeICIC, respectively. As for scheduling, during ABS, only LTE-R eNBs are supposed to schedule the UEs when eICIC is applied. During PRABS, the center UEs served by PS-LTE eNBs will also be scheduled when FeICIC is considered. Many researches have been done on eICIC and FeICIC [15-20], some preferring to schedule both CRE UEs and center UEs of small cells during ABSs/PR-ABSs, while the rest assume that only the CRE UEs are scheduled. According to reference [19], to avoid inefficient utilization of resources during ABS and to achieve better fairness, proportional fair (PF) scheduling is better used for both UEs during ABSs/PR-ABSs.

\subsubsection{SINR and throughput calculation}

The SINRs observed by UE $u$ on PRB $n$ during a normal subframe are given in Eq. (6): 


$$
\left\{\begin{array}{l}
\operatorname{SINR}_{m, u}^{n}=\frac{G_{m, u}^{n} p_{m, u}^{n}}{\sum_{i \in M, i \neq m} I_{i, u}^{n}+\sum_{j \in K} I_{j, u}^{n}+\eta}, u \in U_{M} \\
\operatorname{SINR}_{k, u}^{n}=\frac{G_{k, u}^{n} p_{k, u}^{n}}{\sum_{i \in M} I_{i, u}^{n}+\sum_{j \in K, j \neq k} I_{j, u}^{n}+\eta}, u \in U_{K}
\end{array}\right.
$$

where $p_{m, u}^{n}$ and $p_{k, \mu}^{n}$ are the transmission powers of PSLTE eNBs and LTE-R eNBs on PRB $n$, respectively. The interference received by UE $u$ from neighbor PS-LTE eNB $i$ and neighbor LTE-R eNB $j$ is given as:

$$
\begin{cases}I_{i, u}^{n}=G_{i, u}^{n} p_{i, u}^{n}, & i \in M \\ I_{j, u}^{n}=G_{j, u}^{n} p_{j, u}^{n}, & j \in K\end{cases}
$$

During ABS, the SINR of the UEs served by LTE-R eNBs can be calculated as follows:

$$
\operatorname{SINR}_{k, u}^{n}=\frac{G_{k, u}^{n} p_{k, u}^{n}}{\sum_{j \in K, j \neq k} I_{j, u}^{n}+\eta}, u \in U_{K}
$$

where the interference only comes from other LTE-R eNBs. During PR-ABS, the SINR is expressed as:

$$
\operatorname{SINR}_{k, u}^{n}=\frac{G_{k, u}^{n} l_{k, u}^{n}}{\sum_{i \in M}\left(I_{i, u}^{n} / 10^{\Delta}\right)+\sum_{j \in K, j \neq k} I_{j, u}^{n}+\eta}, u \in U_{K}
$$

where $\Delta$ is the power reduction level in $\mathrm{dB}$ compared to the maximum transmission power during normal subframes. The overall system throughput for eICIC and FeICIC is expressed in Eqs. (10) and (11), respectively.

$$
\begin{aligned}
& R_{\mathrm{eICIC}}=\underbrace{\sum_{k \in K} \sum_{n \in \mathrm{RB}_{\mathrm{np}}^{k}, u \in U_{\mathrm{CK}}} r_{u}^{n}+\sum_{k \in K} \sum_{n \in \in \mathrm{B}_{p}^{k}, u \in U_{K}} r_{u}^{n}} \\
& +\underbrace{\sum_{m \in M} \sum_{\substack{\mathrm{RB}_{\mathrm{fp}}^{m}, u \in U_{\mathrm{CM}} \\
\text { Throughput of }}}^{\sum_{u}^{n}}}_{\text {Throughput of PS-LTE cells }} \\
& R_{\mathrm{FeICIC}}=\underbrace{\sum_{\text {Throuhput of ITE-R cells }}}_{\sum_{k \in K} \sum_{n \in \mathrm{RB}_{\mathrm{np}}^{k}, u \in U_{\mathrm{CK}}} r_{u}^{n}+\sum_{k \in K} \sum_{n \in \mathrm{RB}_{p}^{k}, u \in U_{K}} r_{u}^{n}} \\
& +\underbrace{\sum_{m \in M} \sum_{n \in \mathrm{RB}_{\mathrm{fp}}^{m}, u \in U_{\mathrm{CM}}}^{\text {Throughput of LTE-R cells }} r_{u}^{n}+\sum_{m \in M} \sum_{n \in \mathrm{B}_{\mathrm{rp}}^{m}, u \in U_{M}} r_{u}^{n}}_{\text {Throughput of PS-LTE cells }}
\end{aligned}
$$

\subsection{CS CoMP between LTE-R eNBs}

\subsubsection{Dynamic coordinated muting based coordinated scheduling process}

In this section, a dynamic coordinated muting (DCM)based CS CoMP scheme is introduced among LTE-R eNBs using the LTE Rel. 11 framework [8]. In this paper, the PRBs are allocated by one central scheduler [21]. CoMP UE identification is done in individual eNBs based on UE feedback information. According to the resource allocation and muting decision made in the central scheduler, MCS selection is done in each eNB for the UEs with and without CoMP assistance by coordinated link adaptation, as shown in Fig. 6. Unlike the cell specific muting scheme done elsewhere [22, 23], this paper considers a PRB specific muting scheme.

Figure 7 shows the details of the CS CoMP procedure. All the LTE-R eNBs are considered to be in one CoMP set, and there is one centralized scheduler that will jointly process the information obtained from each LTE$\mathrm{R}$ eNB. To identify which UEs need CoMP assistance and which neighbor cells should provide the assistance, the interference from the neighbor cell should be evaluated using Eq. (12):

$$
\operatorname{SIR}_{u}^{j}>\text { SIR_threshold }
$$

where $\operatorname{SIR}_{u}^{j}$ is the signal-to-interference ratio for UE $u$ and interfering cell $j$. Since LTE-R UE has higher priority than the rest of the UEs served by the LTE-R eNBs, the LTE-R UE will be scheduled first, as shown in Fig. 7, and whenever the LTE-R UE needs CoMP assistance, the neighbor eNB should mute the corresponding RBs. As for the rest of the UEs, there are competition rules. The center CoMP scheduler will randomly choose one eNB to make the scheduling decision based on the PF scheduling rule in Eq. (13) in order to assign PRBs:

$$
u=\arg \max \left(\frac{r_{u}^{a}}{\operatorname{avg} \_t h r_{u}}\right)
$$

where $r_{u}^{a}$ is the rate that can be achieved by UE $u$ on RB $a$, and avg_thr ${ }_{u}$ is the average UE throughput. Then, check the interference measurement results and find out if this UE needs CoMP assistance. If yes, this RB cannot be assigned in the neighbor eNB again, but the other eNBs can still use the RB. Otherwise, the rest of the eNBs can use this RB.

Because each sector is reusing the whole band and it also needs to make scheduling decision over all the RBs. We assume that there are total $L$ sectors, and for each sector $N$ RBs and $K$ UEs are considered. According to Fig. 7, for each RB $i$, the selected sector $S_{v}$ needs to make scheduling decision which is to select an UE $j$. Since the UE selection is based on PF metric in Eq. (13), at most, $K-1$ times of comparisons need to be made. If the RB is allocated to the UE who does not need CoMP assistance then make the scheduling decision for the neighbor sectors of $S_{v}$, which is $S_{v-1}$ and $S_{v+1}$. It should be noted that the RB can only be scheduled to that UEs who do not need CoMP assistance so if there are UEs who need CoMP assistance in $S_{v-1}$ and $S_{v+1}$, making the 


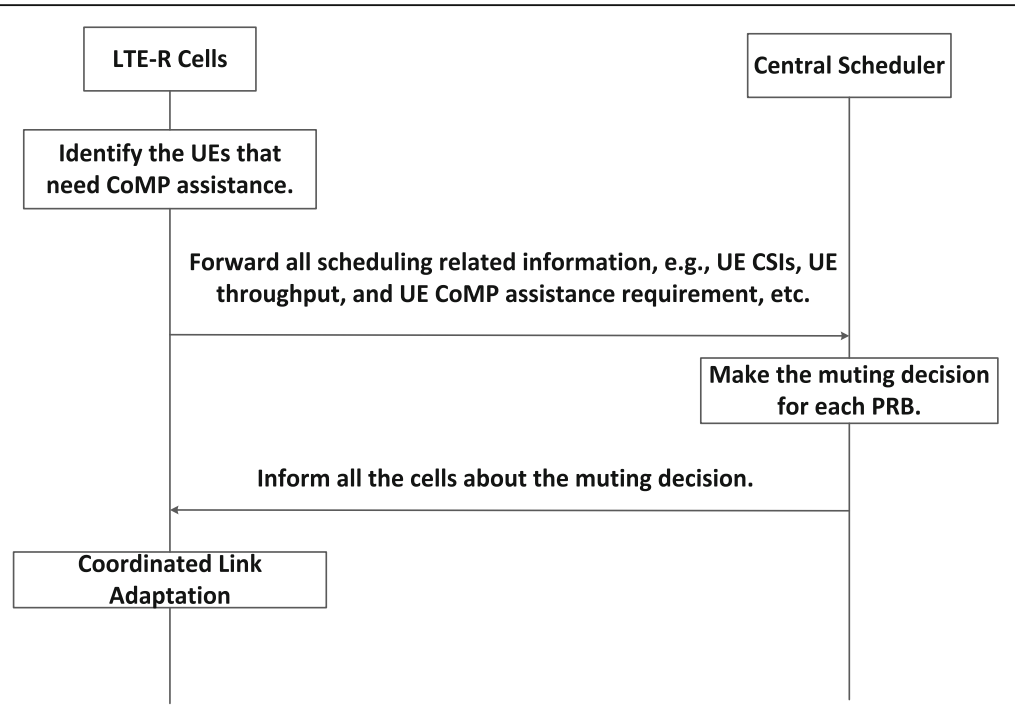

Fig. 6 Centralized CS CoMP for LTE-R network

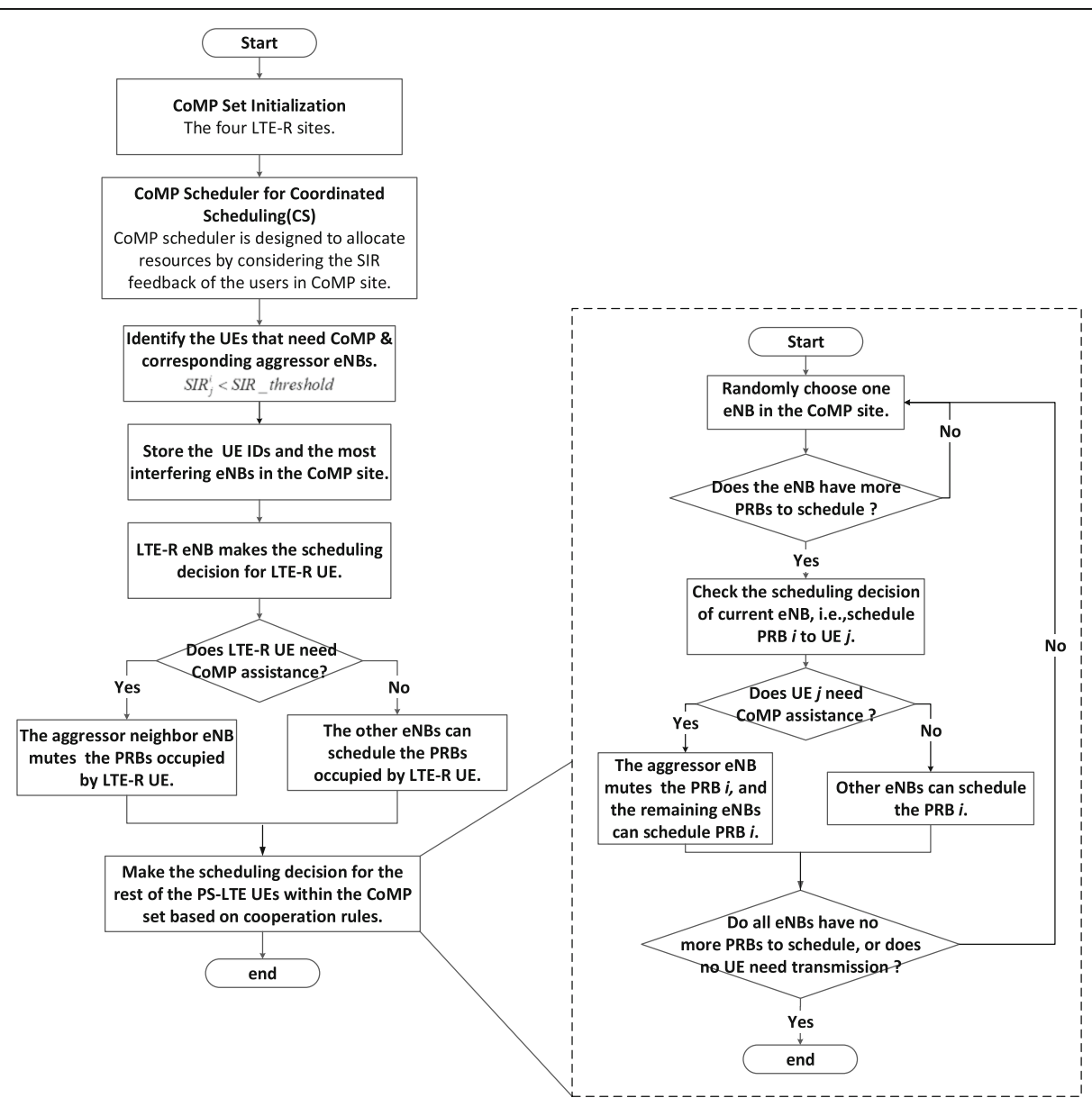

Fig. 7 Flow chart for CS CoMP between LTE-R eNBS 
scheduling decision on RB $i$ will need less than $K-1$ times comparisons. If the sector $S_{v}$ allocate the RB to the UE who needs CoMP assistance, $S_{v-1}$ and $S_{v+1}$ will mute their RB $i$ and $S_{\mathrm{v}-2}$ and $S_{v-3}$ will then schedule the RB $i$. Considering that there are no neighbors of them that are using RB $i$, they can allocate it to either UE who needs CoMP assistance or not. This loop will stop until all the sectors made their scheduling decision of RB $i$. For each $\mathrm{RB}$, at most, $L$ sectors should make their scheduling decision and each decision takes at most $K-1$ times comparison. Besides, once an RB is allocated, the bits that it can be used to transmit for the corresponding UE will be got through table searching and will be subtracted from the total bits that UE needs to transmit. Considering all the calculation steps mentioned above, the complexity which is expressed by $O$ of the coordinated scheduling procedures can be calculated as follows:

$$
O(\operatorname{LN}(K-1)+L N) \approx O(\mathrm{LNK})
$$

\subsubsection{Multiple CSI processes based coordinated link adaptation}

It should be noted that if the neighbor cell mutes certain resources to support the UE that needs CoMP assistance, the MCS level should be selected based on the hypothesis of no interference from the neighbor cell. Hence, higher spectral efficiency for the corresponding RBs can be achieved.

Since the CoMP set only includes the LTE-R eNBs, LTE-R eNBs are located along the train line for each UE served by LTE-R eNBs; at most, one neighbor LTE-R cell will cause high interference to the UE. Therefore, among all the LTE-R sectors, each cell edge UE only needs one LTE- $\mathrm{R}$ sector to mute corresponding RBs and whether other cells mute the same RBs will not make much difference for the UE. In order to identify that if UE needs CoMP assistance from neighbor cell and executes adaptive link adaptation when the neighbor cell mutes certain RBs then each UE needs channel state information (CSI) under two hypothesizes. One is the CSI information when UE gets interference from the neighbor cell; another is the CSI when UE gets no interference from the neighbor cell. Hence, each UE is configured with two channel state information (CSI) processes [14]. CSI process -0 is configured to get normal CQI indexes, where all the cells are transmitting on the same transmitting resource elements. CSI process- 1 reflects the benefit obtained by the UE if the strongest interfering cell is muted. CSI process- 1 can be achieved by allowing the neighbor LTE-R eNBs to use orthogonal resource elements for reference signal transmission. For the UEs supported by CoMP assistance, the MCS level selection is performed based on the channel quality measured by
CSI process-1. In addition, these two CSI processes are also used to support the UE-received interference evaluation in Eq. (12).

\section{Performance evaluation of the interference management schemes}

\subsection{Simulation environment and assumptions}

To evaluate the performance of the scenario without RAN sharing and LTE-R RAN sharing by PS-LTE UEs, we perform the SLS. Moreover, the performance of interference management schemes is verified for the coexistence of PS-LTE and LTE-R networks. The main SLS parameters are given in Table 1. Instead of full buffer traffic, the realistic traffic models are considered, e.g., voice over internet protocol (VoIP) and video. For LTE-R UE, only VoIP is used to model the traffic of the train control signal. In addition, we consider three ABSs/PR-ABSs per

Table 1 System level simulation parameters

\begin{tabular}{|c|c|}
\hline Parameter & Value \\
\hline Carrier frequency (DL) & $778 \mathrm{MHz}$ \\
\hline $\begin{array}{l}\text { Bandwidth (PS-LTE } \\
\text { eNB/LTE-R eNB) }\end{array}$ & $10 \mathrm{MHz}$ \\
\hline No. of PRBs & 50 \\
\hline RB bandwidth & $180 \mathrm{KHz}$ \\
\hline No. of PS-LTE eNBs & $\begin{array}{l}21 \text { sectors (1-tier, } 7 \text { sites) (only } 3 \text { inner } \\
\text { sectors are the region of interest) }\end{array}$ \\
\hline No. of LTE-R eNBs & Maximum 2 eNBs/sector beside the railway \\
\hline Inter-eNB distance & $\begin{array}{l}\text { PS-LTE eNBs, } 4 \text { km } \\
\text { LTE-R eNBs, } 1 \text { km }\end{array}$ \\
\hline No. of UEs/sector & $\begin{array}{l}\text { PS-LTE UES, } 40 \\
\text { LTE-R UE, } 1 \\
\text { (1 terminal for train control) }\end{array}$ \\
\hline Transmission power & $\begin{array}{l}\text { PS-LTE, } 46 \mathrm{dBm} \\
\text { LTE-R, } 43 \mathrm{dBm}\end{array}$ \\
\hline Maximum antenna gain & $\begin{array}{l}\text { PS-LTE, } 15 \mathrm{dBi} \\
\text { LTE-R, } 17 \mathrm{dBi}\end{array}$ \\
\hline Minimum coupling loss & $70 \mathrm{~dB}$ \\
\hline Noise spectral density & $-174 \mathrm{dBm} / \mathrm{Hz}$ \\
\hline Path loss model & Rural macro (3GPP TR 36.837) \\
\hline Shadowing & $\begin{array}{l}\text { Log-normal distribution (mean } 0 \mathrm{~dB} \text {, st. } \\
\text { dev. } 6 \mathrm{~dB} \text { ) } \\
\text { (Correlation b/w eNBs/sectors, } 0.5 / 1 \text { ) }\end{array}$ \\
\hline Fast fading & $\begin{array}{l}\text { PS-LTE: winner II (D1_rural macro) } \\
\text { LTE-R: winner II (D2a—rural moving networks) }\end{array}$ \\
\hline UE mobility & $\begin{array}{l}\text { PS-LTE UE, } 3 \mathrm{Km} / \mathrm{h} \\
\text { LTE-R UE, } 250 \mathrm{Km} / \mathrm{h}\end{array}$ \\
\hline Transmission modes & $\mathrm{SISO}(1 \times 1)$ \\
\hline Effective SINR & MIESM \\
\hline UE receiver & Zero forcing \\
\hline Traffic models & $\begin{array}{l}\text { PS-LTE: VolP (80\%), video (20\%) } \\
\text { LTE-R: VoIP }\end{array}$ \\
\hline
\end{tabular}


frame for eICIC and FeICIC. Moreover, the bias value for LTE-R CRE is $6 \mathrm{~dB}$ for both eICIC and FeICIC. To avoid high interference to UEs in the CRE area, a $7 \mathrm{~dB}$ power reduction can be used for PS-LTE eNB to transmit the data for the center UEs during PR-ABSs.

\subsection{Simulation results and discussion}

In Fig. 8, the SINR distribution under the RAN sharing environment is given. The cell selection for RAN sharing is done based on Eq. (15) below, where $\operatorname{RSRP}_{u}^{z}$ is the reference signal receiving power of UE $u$ from eNB $z$. We can see that LTE-R cells occupy almost half of the coverage of the two sectors of the center PS-LTE eNB, which implies that under uniform distribution of PS-LTE UEs, around half of the UEs will access the LTE-R eNBs. It also shows that LTE-R UE is moving along the track and usually enjoys better channel quality by connecting to LTE-R eNBs. However, when LTE-R UE moves to the cell edge, also close to the PS-LTE eNB, it can suffer high interference from the PS-LTE eNB.

$$
\text { Serving_eNB_ID }{ }_{u}=\underset{z \in(M \cup K)}{\operatorname{argmax}}\left(\operatorname{RSRP}_{u}^{z}\right)
$$

Figure 9 shows the SINR of LTE-R UE at different positions, where the $x$-axis indicates the $y$-coordinate of the LTE-R UE position, while the $x$-coordinate is assumed to be constant, because LTE-R UE moves along the track between LTE-R eNBs. We can see that while at the cell edge between neighbor sectors and at the cell edge between neighbor sites, LTE-R UE suffers high interference and bad channel conditions. At the range of $200 \sim 300 \mathrm{~m}$, there are more chances that the LTE$\mathrm{R}$ UE-received SINR goes lower than $-5 \mathrm{~dB}$ because this area is not only the cell edge between neighbor
LTE-R sites but is also close to the center PS-LTE eNB, as marked in Fig. 8.

Figure 10 shows the SINR of LTE-R UE when CS CoMP and eICIC are applied, and we can see that the cell edge SINR of LTE-R UE has greatly increased due to the interference mitigation at the cell edge by CS CoMP and eICIC. It should be noted that by using eICIC scheme, LTE-R UE can be served with better channel quality than FeICIC because there is no interference from PS-LTE eNBs during ABSs. However, the curve for FeICIC and CS CoMP is similar to Fig. 10, since most of the time, SINR is measured during normal subframes, while there are only $30 \%$ ABSs. Besides, the major improvement is achieved by CS CoMP between neighbor LTE-R eNBs.

As shown in Fig. 11, that channel qualities for UEs are greatly improved using RAN sharing. When the SINR threshold is $-5 \mathrm{~dB}$, around $20 \%$ of PS-LTE UEs will be in outage without RAN sharing. The outage probability is given in Eq. (16). For the scenario with LTE-R RAN sharing by PS-LTE UEs, only around 3\% of the PS-LTE UEs are in outage. This is due to the conversion of the high-interference source to the desired signal, while at the same time, the previous weak desired signal will become part of the interference, which improves the signal power and decreases the interference power, as shown in Fig. 12. In the scenarios with eICIC/FeICIC and CS CoMP, the channel quality is significantly improved and at the $2 \mathrm{~dB}$ threshold, the outage probabilities have decreased to $6.1 \%$ and $9.4 \%$, respectively, compared to the $51.5 \%$ for the scenario only with RAN sharing, due to the benefits of ABS/PR-ABS and CS CoMP

$$
P(\text { outage })=1-P\left(\text { SINR }>\text { SINR } \_ \text {Threshold }\right)
$$

Figure 13 shows the improvement in the throughput of PS-LTE UEs using RAN sharing. At 50\% of cumulative distribution function (CDF) curve, the UE throughput has

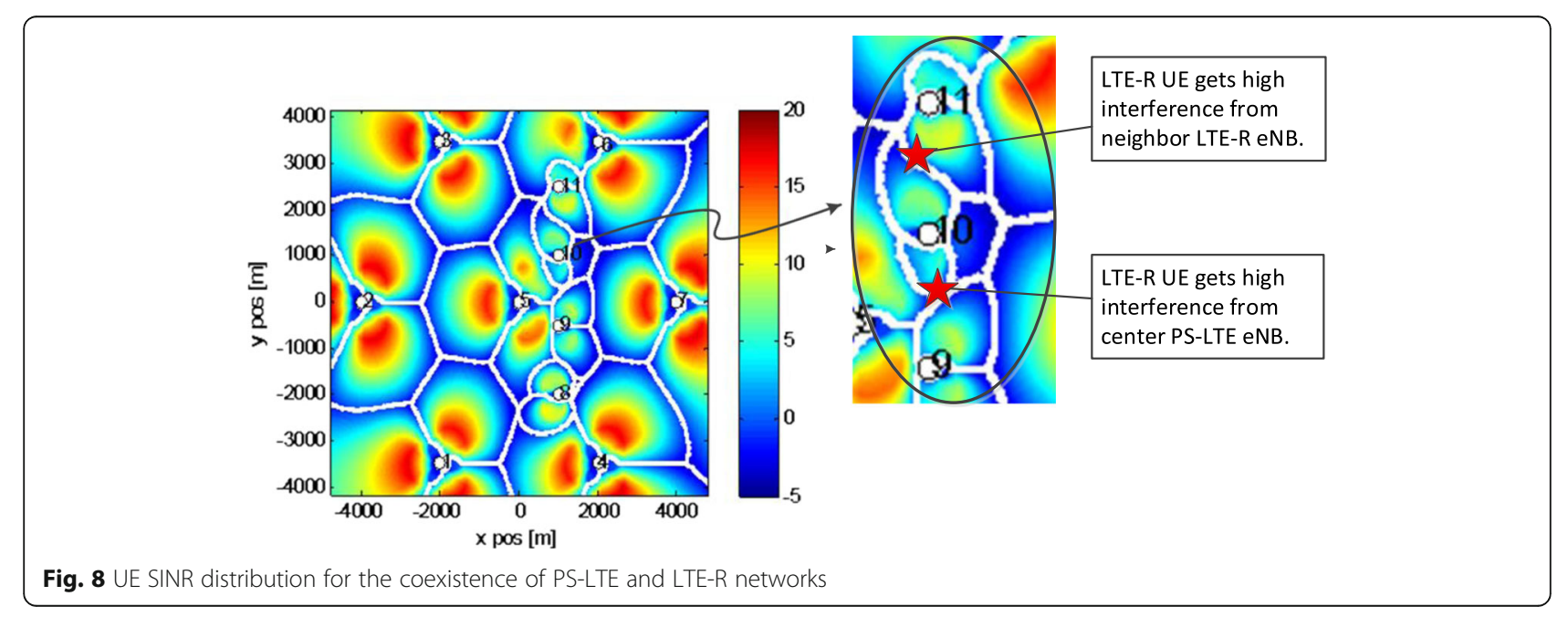




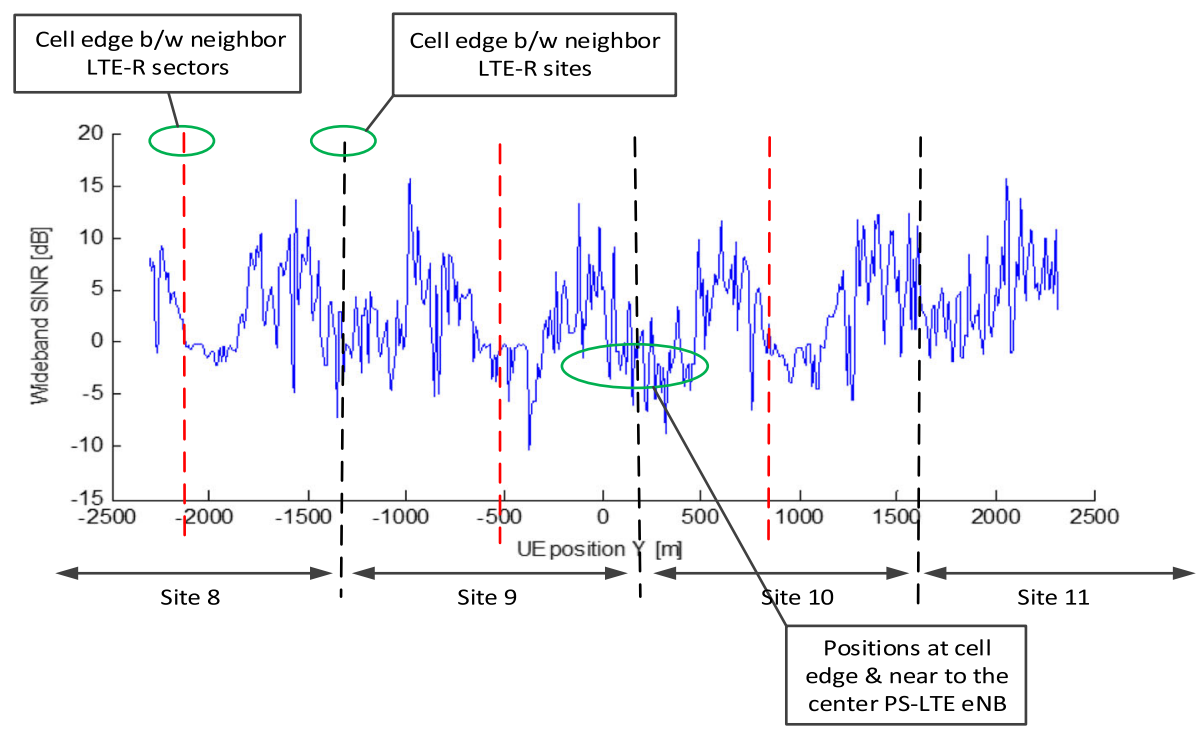

Fig. 9 LTE-R UE SINR without interference management schemes

increased by around 7.3\%. There are two reasons for the improvement. The first is that the channel quality for PSLTE UEs is getting better. Another is that more resources are available for PS-LTE UEs with RAN sharing. When a PS-LTE eNB is under high load, the LTE-R eNBs can offload PS-LTE users using RAN sharing and offer better services to these users. In the scenario with RAN sharing and eICIC, the edge throughput performance is further improved because of the CRE and ABS. Due to CRE, more PS-LTE UEs can be offloaded to the LTE-R eNB. Since there are two LTE-R eNBs (four sectors) per PS-LTE sector reusing the same band as the PS-LTE eNB, many PS-LTE UEs should be allowed to access the services provided by LTE-R eNBs. Then, with ABS, these UEs can even enjoy better channel conditions, compared to the channel conditions while being served by PS-LTE eNBs. Applying FeICIC and CoMP under the case of RAN sharing achieves the best performance among all the scenarios, because using PR-ABS, the resource utilization of the PSLTE eNBs is enhanced, compared to the zero-power ABS for eICIC.

Figure 14 shows the PS-LTE UE SINR to spectral efficiency performance, where the $x$-axis is the UE SINR and the $y$-axis is the UE spectral efficiency in effective data bits per channel use (bit/cu). The value of spectral efficiency is determined by MCS level and BLER. By

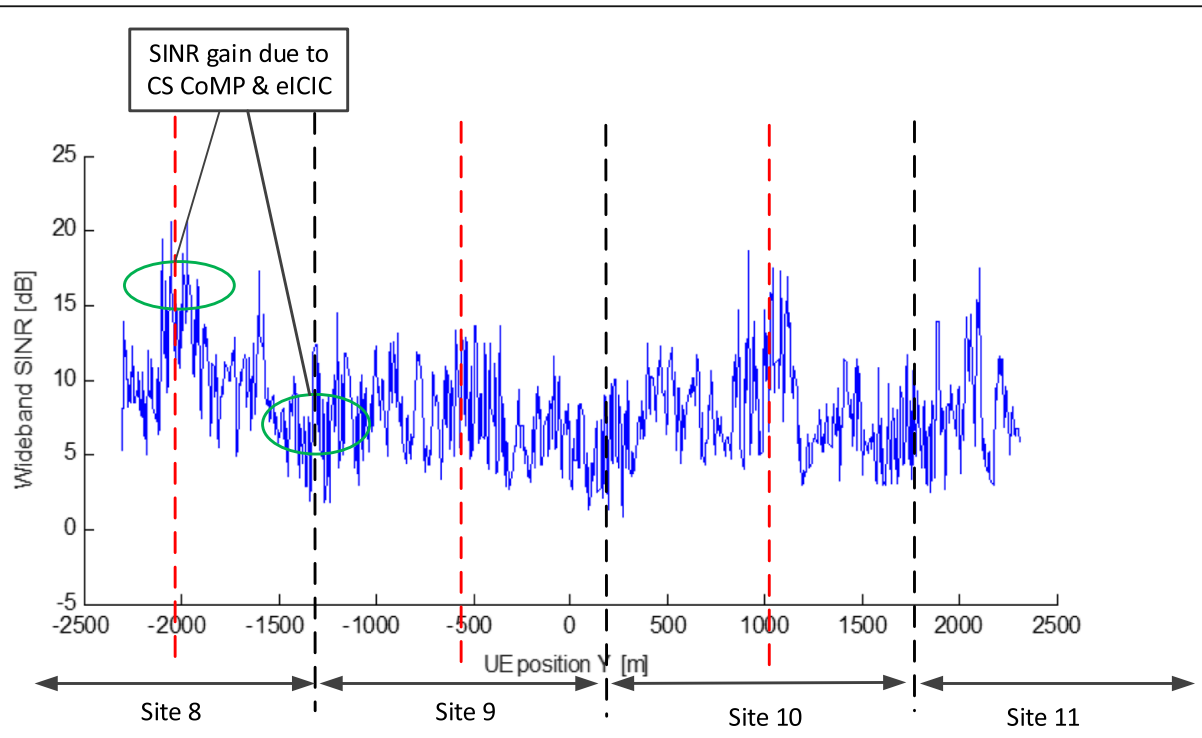

Fig. 10 LTE-R UE SINR with eICIC and CS COMP 


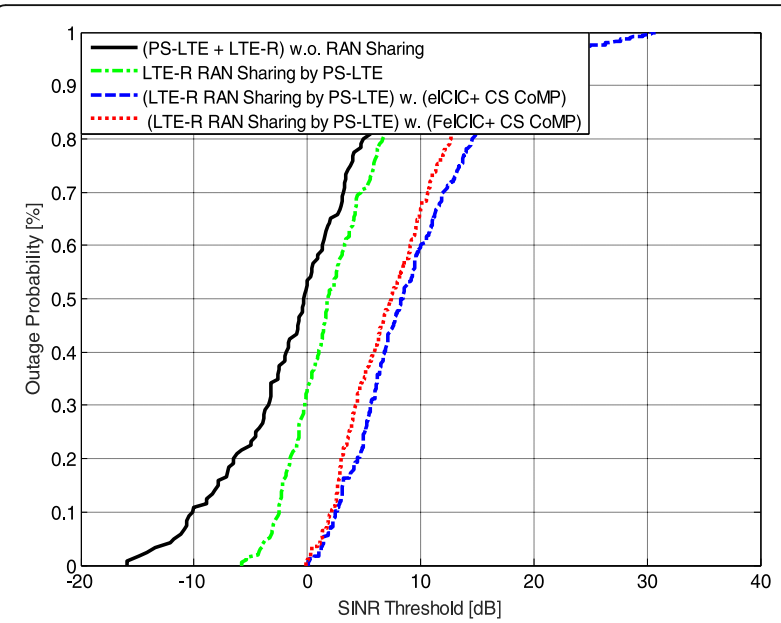

Fig. 11 UE outage probability

using the AMC scheme abstracted in the PHY layer, the MCS level is selected based on the policy to guarantee BLER within $10 \%$. In the scenario with eICIC, the maximum spectral efficiency is higher than other scenarios because the muting of PS-LTE eNBs and CS CoMP results in better channel quality; then, higher MCS levels can be selected for the UEs. In the scenario with FeICIC, the maximum spectral efficiency is lower than that with eICIC due to the data transmission of PS-LTE eNBs during power-reduced $A B S$, which gives interference to the UEs served by LTE-R eNBs. Overall, SINR to spectral efficiency curves for all the scenarios almost overlap, because the same AMC scheme is used in the LTE network for all the UEs.

\section{Conclusions}

This paper introduces scenarios for the coexistence of PS-LTE and LTE-R networks and evaluates the system

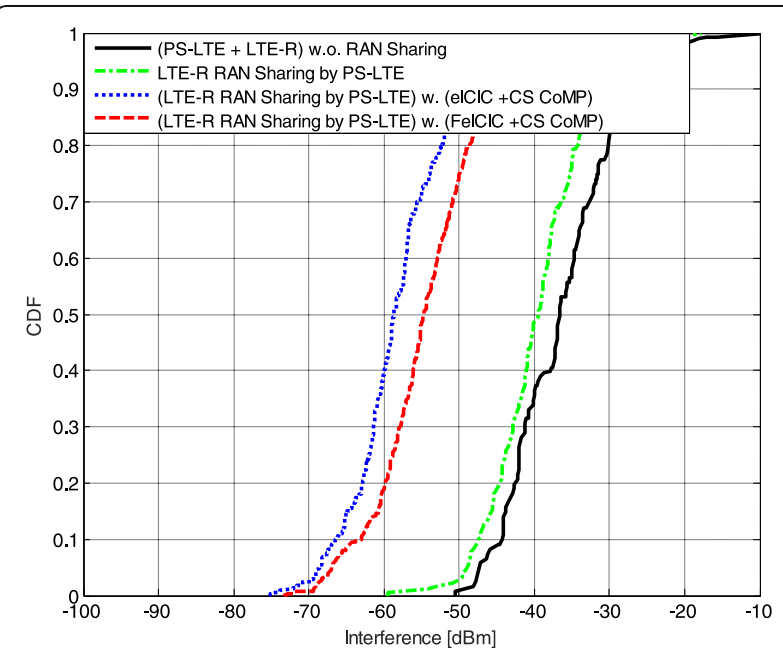

Fig. 12 UE Rx interference

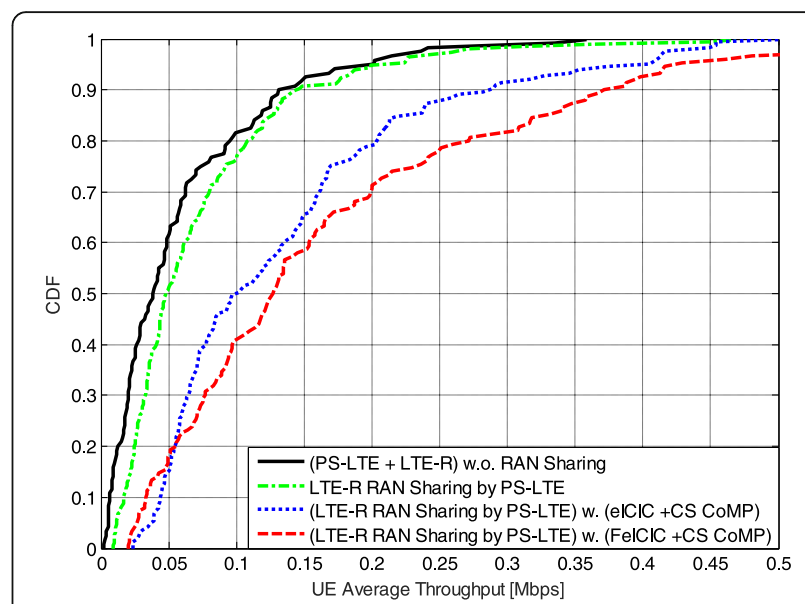

Fig. $13 \cup E$ throughput

performance under the cases of non-RAN sharing and LTE-R RAN sharing by PS-LTE UEs. Simulation results show that there is around a $17 \%$ reduction in the outage probability and a $7 \%$ improvement of 50 th percentile UE throughput using RAN sharing. To improve the reliability of the train control signal and to further enhance PSLTE UE performance, time-domain eICIC and FeICIC along with CoMP are applied. From the results shown in section 5, with an SINR threshold of $2 \mathrm{~dB}$, the outage probabilities of scenarios 3 and 4 decrease to $6.1 \%$ and $9.4 \%$, respectively, compared to the $51.5 \%$ in scenario 2 without any interference management schemes. The throughput significantly increases due to the load balancing by CRE and interference coordination by using $\mathrm{ABS} / \mathrm{PR}-\mathrm{ABS}$ and CS CoMP for the cell edge UEs. Because there are still data transmitted from PS-LTE eNBs during PR-ABS, scenario 4 shows the best throughput performance. In addition, by using the interference

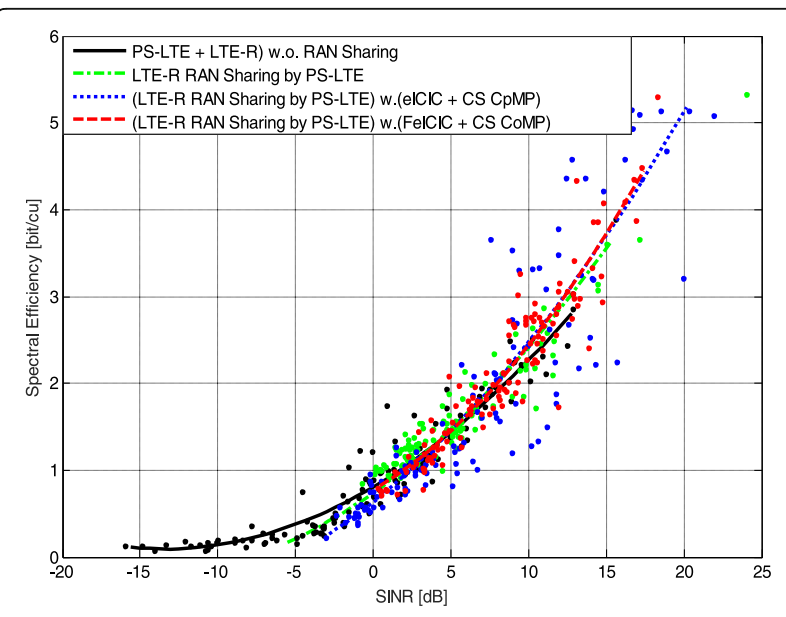

Fig. $14 \cup E$ SINR to spectral efficiency 
coordination schemes, the channel quality for LTE-R UE shows remarkable improvement, especially for scenario 3, due to the complete muting of PS-LTE eNBs during ABS.

\section{Acknowledgements}

This research was supported by the MSIP (Ministry of Science, ICT and Future Planning), Korea, under the ITRC (Information Technology Research Center) support program (IITP-2016-H8501-15-1019) supervised by the IITP (Institute for Information \& communications Technology Promotion).

\section{Competing interests}

The authors declare that they have no competing interests.

Received: 29 June 2016 Accepted: 3 February 2017

Published online: 20 February 2017

\section{References}

1. J.K. Choi, H. Cho, K.H. Kim, Challenges of LTE high-speed railway network to coexist with LTE public safety network, in Proceedings of IEEE International Conference on Advanced Communication Technology (IEEE, Seoul, Korea, 2015), pp. 543-547

2. T Guo, R Arnott, Active LTE RAN sharing with partial resource, in Proceedings of Vehicular Technology Conference, 2013, pp. 2-5

3. 3GPP, R1-105081: Summary of the description of candidate elClC solutions, in 3GPP TSG RAN WG1 Meeting 62 (3GPP, Madrid, Spain, 2010)

4. 3GPP, Evolved Universal Terrestrial Radio Access (E-UTRA) and Evolved Universal Terrestrial Radio Access Network (E-UTRAN); Overall descriptions (Release 12), 3GPP TS 36.300 (2015)

5. 3GPP, Technical Specification Group Radio Access Network; Coordinated multi-point operation for LTE physical layer aspects (Release 11), 3GPP TR 36.819 (2011)

6. N. Naganuma, S. Nakazawa, S. Suyama, Adaptive control CRE technique for elCIC in HetNet, in Proceedings of IEEE International Conference on Ubiquitous and Future Networks (ICFUN) (IEEE, Vienna, 2016), pp. 4-6

7. H Zhou, YS Ji, XY Wang, elClC configuration algorithm with service scalability in heterogeneous cellular networks. IEEE/ACM Trans Networking. PP(99), 1-16 (2016)

8. A Merwaday, I Guvenc, Optimization of FelCIC for energy efficiency and spectrum efficiency in LTE-advanced HetNets. Electron Lett 52(11), 982-984 (2016)

9. C Huang, QB Chen, L Tang, Hybrid inter-cell interference management for ultra-dense heterogeneous network in 5G. Sci China Inf Sci 59, 082305 (2016). doi:10.1007/s11432-016-5556-2

10. 3GPP, Technical Specification Group Radio Access Network; Public safety broadband high power user equipment (UE) (Release 11), 3GPP TR 36.837 (2012)

11. 3GPP, Evolved Universal Terrestrial Radio Access (E-UTRA); Radio frequency (RF) system scenarios (Release 8), 3GPP TR 36.942 (2014)

12. H. Claussen, Efficient modeling of channel maps with correlated shadow fading in mobile radio systems, in Proceedings of IEEE 16th International Symposium on Personal Indoor and Mobile Radio Communications (PIMRC) (IEEE, Berlin, Germany, 2005), pp. 512-516

13. L. Hentila, P. Kyosti, M. Alatossava, MATLAB implementation of the WINNER phase II channel model, v1.1 (Sept. 30, 2007), http://projects.celtic-initiative org/winner+/WINNER2-Deliverables/D1.1.2v1.1.pdf. Accessed 30 Sept 2007

14. 3GPP, Technical Specification Group Radio Access Network; Physical layer procedures, 3GPP TS 36.213 (2016)

15. Z. Hanzaz, H.D. Schotten, Analysis of effective SINR mapping models for MIMO OFDM in LTE system, in Proceedings of IEEE International Wireless Communications and Mobile Computing Conference (IEEE, Italy, Sardinia, 2013), pp. 1-5

16. S Deb, P Monogioudis, J Miernik, Algorithms for enhanced inter-cell interference coordination (elCIC) in LTE HetNets. IEEE/ACM Trans. Networking 22(1), 137-150 (2014)

17. J.B. Abderrazak, M. Sfar, H. Besbes, Fair scheduling and dynamic ICIC for multicellular OFDMA systems, in Proceedings of IEEE International Conference and Workshop on the Network of the Future (NOF) (IEEE, Paris, France, 2014) pp. 1-5

18. Y. Ikeda, S. Okasaka, M. Hoshino, Proportional fair-based joint optimization of cell association and inter-cell interference coordination for heterogeneous networks, in Proceedings of IEEE 80th Vehicular Technology Conference (VTC Fall) (IEEE, Vancouver, Canada, 2014), pp. 1-5

19. H.T. Du, W.A. Zhou, X.Q. Lu, An optimized resource allocation and CoMP based interference coordination scheme for LTE-A Het-Net, in Proceedings of IEE 9th International Conference on Broadband and Wireless Computing, Communication and Applications (BWCCA) (IEEE, Guangdong, China, 2014), pp. 207-211
20. K. Somasundaram, Proportional fairness in LTE-advanced heterogeneous networks with eICIC, in Proceedings of IEEE Vehicular Technology Conference (VTC Fall), (IEEE, Las Vegas, NV, America, 2013), pp. 1-6

21. X.Y. Wang, B. Mondal, A. Ghosh, Coordinated scheduling and network architecture for LTE macro and small cell deployments, in Proceedings of IEEE International Conference on Communication Workshops (ICC), (IEEE, Sydney, Australia, 2014), pp. 604-609

22. G. Nardini, G. Stea, A. Virdis, Effective dynamic coordinated scheduling in LTE-advanced networks, in Proceedings of IEEE European Conference on Networks and Communications (EuCNC), (IEEE, Bologna, Italy, 2014), pp. 1-5

23. R. Agrawal, A. Bedekar, N. Arulselvan, Centralized and decentralized coordinated scheduling with muting, in Proceedings of IEEE Vehicular Technology Conference (VTC Spring), (IEEE, Seoul, Korea, 2014), pp. 1-5

\section{Submit your manuscript to a SpringerOpen ${ }^{\circ}$ journal and benefit from:}

- Convenient online submission

- Rigorous peer review

- Immediate publication on acceptance

- Open access: articles freely available online

- High visibility within the field

- Retaining the copyright to your article

Submit your next manuscript at $>$ springeropen.com 